\title{
Service and Education: The Association Between Workload, Patient Complexity, and Teaching on Internal Medicine Inpatient Services
}

\author{
Temple A. Ratcliffe, $M D^{1,2}$, Meghan A. Crabtree, $M S^{3}$, Raymond F. Palmer, $P h D^{7}$, \\ Jacqueline A. Pugh, MD ${ }^{1,2}$, Holly J. Lanham, PhD, MBA ${ }^{7,2,4}$, and Luci K. Leykum, MD, MPH, MSc ${ }^{1,2,4}$
}

'University of Texas Health Science Center at San Antonio, San Antonio, TX, USA; ${ }^{2}$ South Texas Veterans Health Care System, San Antonio, TX, USA; ${ }^{3}$ University of Texas at San Antonio, San Antonio, TX, USA; ${ }^{4}$ University of Texas at Austin, Austin, TX, USA.

\begin{abstract}
BACKGROUND: Attending rounds remain the primary venue for formal teaching and learning at academic medical centers. Little is known about the effect of increasing clinical demands on teaching during attending rounds.
\end{abstract}

OBJECTIVE: To explore the relationships among teaching time, teaching topics, clinical workload, and patient complexity variables.

DESIGN: Observational study of medicine teaching teams from September 2008 through August 2014. Teams at two large teaching hospitals associated with a single medical school were observed for periods of 2 to 4 weeks.

PARTICIPANTS: Twelve medicine teaching teams consisting of one attending, one second- or third-year resident, two to three interns, and two to three medical students.

MAIN MEASURES: The study examined relationships between patient complexity (comorbidities, complications) and clinical workload variables (census, turnover) with educational measures. Teams were clustered based on clinical workload and patient complexity. Educational measures of interest were time spent teaching and number of teaching topics. Data were analyzed both at the daily observation level and across a given patient's admission.

KEY RESULTS: We observed 12 teams, 1994 discussions (approximately $373 \mathrm{~h}$ of rounds) of 563 patients over 244 observation days. Teams clustered into three groups: low patient complexity/high clinical workload, average patient complexity/low clinical workload, and high patient complexity/high clinical workload. Modest associations for team, patient complexity, and clinical workload variables were noted with total time spent teaching $(9.1 \%$ of the variance in time spent teaching during a patient's admission; $F[8,549]=6.90, p<0.001)$ and number of teaching topics $(16 \%$ of the variance in the total number of teaching topics during a patient's admission; $F[8,548]=$ $14.18, p<0.001$ ).

CONCLUSIONS: Clinical workload and patient complexity characteristics among teams were only modestly associated with total teaching time and teaching topics.

KEY WORDS: medical education; medical education-graduate; medical education-undergraduate; hospital medicine.

Received June 13, 2017

Revised October 31, 2017

Accepted December 28, 2017

Published online February 1, 2018
J Gen Intern Med 33(4):449-54

DOI: $10.1007 / \mathrm{s} 11606-017-4302-9$

(c) Society of General Internal Medicine (outside the USA) 2018

\section{INTRODUCTION}

Despite changes in medical education, teaching rounds remain a core component of learner experiences. ${ }^{1-3}$ Rounds are the main venue for faculty to teach by reviewing resident plans, assessing learners' knowledge, and offering teaching points that relate to patients on the team or within the system in which they work. However, numerous factors have led to an evolution of teaching rounds. Rounds are now characterized by a blending of work rounds and traditional teaching rounds and are typically around $2 \mathrm{~h}$, much of which is spent in hallways or team rooms. ${ }^{2,}{ }^{3}$ Teaching still occurs but tends to focus on patient care plans and diagnostic study review rather than physical exam skills or other medical topics. ${ }^{2}$

While work-hour reforms have significantly impacted rounds, other national trends have arguably had at least as significant an impact. ${ }^{4}$ Increasing emphasis on productivity, ${ }^{5,6}$ the advent of hospital medicine as a specialty, ${ }^{7,8}$ the introduction of the electronic medical record, ${ }^{9}$ pressure to reduce length of stay and increase throughput, and an increased emphasis on quality and safety, as well as patient satisfaction, have all led to myriad changes in academic inpatient services. These in turn have given rise to increasing concern regarding the duration, quality, and content of rounds, prompting some to propose that rounds would benefit from redesign, with numerous suggestions put forth for improving the educational experience. ${ }^{10-12}$

Understanding the factors that impact teaching is a vital component for effective optimization of contemporary teaching rounds. In our view, this requires exploring the relationships between patient complexity, clinical workload, and teaching. In addition, a better understanding of the content of rounds - what topics specifically are being discussed-is essential. We conducted a comprehensive observational study of attending rounds of inpatient teams at two large teaching hospitals, and explored the relationships among teaching time and census, patient acuity, and other variables. This study contributes to the literature on factors associated with teaching during rounds, and quantification and categorization of teaching topics discussed. 


\section{METHODS}

As part of a series of studies examining relationships and sensemaking ${ }^{13-15}$ among inpatient medicine teams, we analyzed team data from a single residency program at two large teaching hospitals (an urban county hospital and a Department of Veterans Affairs [VA] hospital). A complete description of the methods involving the first 11 teams observed has been published elsewhere. ${ }^{13,15}$ Briefly, researchers observed 12 inpatient medicine teams, taking detailed field notes of all teaching topics covered. Teams were purposefully sampled to obtain a broadly representative group based on physician characteristics and time of year. The 12th team was the first team in a follow-up study and was also identified based on the same criteria used for the initial teams. ${ }^{14}$ Patients who were readmitted were counted as novel encounters for the purpose of data analysis. The 12 inpatient teams were observed from September 2008 through August 2014 for 2 to 4 weeks, with the length of observation dependent on whether the attending was with the team for 2 or 4 weeks. All days were observed with the exception of weekends/holidays when the team did not conduct rounds in person, but instead the attending reviewed patients with the resident by phone, or days that another attending covered. If a team's attending changed during observation and all other team members remained, the team was renumbered with a letter designation (e.g., team 4a became $4 \mathrm{~b}$ when the attending switched). All observed rounds were audio-recorded for the purpose of field note validation and for timing aspects of rounds described below.

While the original focus of our work was on relationships and sensemaking, data used for this analysis were prospectively collected as part of the original protocol. We planned on collecting data regarding teaching and workload, as we thought that these variables could be associated with how team members related to each other and made sense of their tasks. Workload was a covariate in our prior published work. ${ }^{15}$

This study was approved by the University of Texas Health Science Center at San Antonio (UTHSCSA) Institutional Review Board, the South Texas Veterans Health Care System (STVHCS) Research and Development Committee, and the University Health System (UHS) Research Committee.

\section{Teaching Topics}

While taking field notes, three researchers identified when teaching topics were being discussed, took notes on them, and noted their duration. The process for assessing concordance and agreement was described in prior studies, and entailed two observers rounding together on each team and comparing notes daily until $90 \%$ concordance on teaching topics was reached. ${ }^{13,15}$ The notes were then re-analyzed by one investigator (TR), who categorized teaching topics using the American Board of Internal Medicine (ABIM) certification blueprint ${ }^{16}$ as a framework.

\section{Statistical Analysis}

To explore relationships between patient complexity, clinical workload, and educational measures, we examined the effects of patient complexity and clinical workload on both time spent teaching and number of teaching topics. We measured these associations 1) aggregated across observation days for each team, and 2) aggregated across days for each individual patient's admission. By analyzing data across each patient's hospitalization (in addition to observation day), we were able to capture additional teaching variability.

Patient complexity variables of interest were measures of comorbidities and complications. Patient-related data were taken from recordings, followed by chart review of each patient. Comorbidity variables (Comorbidity Score, Average Comorbidities, Comorbidities) were derived from Charlson-Deyo scores. ${ }^{17}$ Complication variables (Daily Complications, Total Complications, Complications of other patients) were derived from instances where a team member noted that the patient had clinically worsened since the last rounds. Clinical workload variables included measures of daily census (Average Daily Census, Daily Census) and patient turnover (Turnover, Daily Turnover). Both patient complexity and clinical workload variables were analyzed across observation days for each team and across each individual patient's admission. By analyzing our data across two levels (observation days and teams), we were able to examine impacts of variables for a given patient, and for other patients cared for by the team, during a given patient's admission.

To explore the association between team characteristics and educational measures, we used a simple cluster analysis technique to organize our 12 teams into three clusters based on similarities and differences in clinical workload and patient complexity. For this analysis, we used observation days as the unit of measure. Specifically, we computed an Overall Daily Workload variable by summing Daily Turnover and Daily Census, and an Overall Patient Complexity variable by summing Comorbidities and Daily Complications. Following their computation, Overall Daily Workload and Overall Patient Complexity variables were standardized $(M=0, S D=1)$. Thus, $Z$-score comparisons $\left(Z_{\text {low }} \leq-0.5, Z_{\text {high }} \geq 0.5\right.$, and $-0.5<Z_{\text {average }}<0.5$ ), as well as scatter plots of the two variables with cases grouped by team, were examined for clustering patterns (see Fig. 1). This allowed us to explore differences between clusters with regard to patient complexity and clinical workload that would otherwise not have been possible due to our relatively small number of teams.

Finally, we conducted two ordinary least-squares hierarchical regression analyses with the three clusters to examine the association between the above-described predictors and our educational measures, using the patient as the unit of analysis.

\section{RESULTS}

Across 12 inpatient teams, we observed 1994 discussions (approximately $373 \mathrm{~h}$ of rounds) of 527 different patients over 


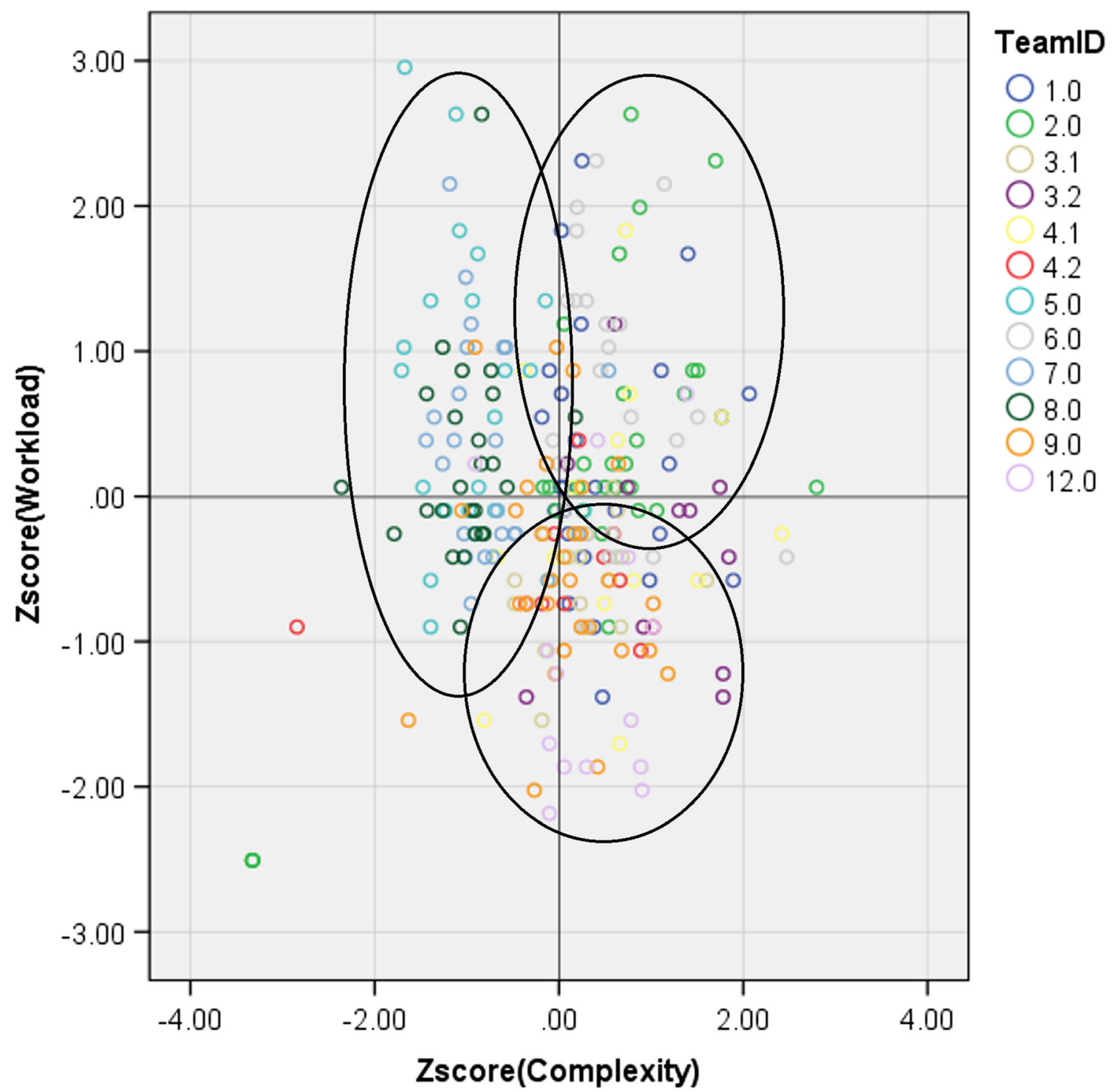

Figure 1 Team clusters of patient complexity by workload.

the course of 244 observation days. Thirty-six patients were readmitted to the same teams during the observation period (and were analyzed as new admissions), bringing the total number of patients to 563 .

Nine of the 12 teaching faculty were men. Faculty experience since completion of residency ranged from 1 to 30 years. There were four assistant professors, three associate professors, and five full professors.

The duration of rounds varied widely, from $20 \mathrm{~min}$ to $4 \mathrm{~h}$ $54 \mathrm{~min}$. The mean duration across teams was $1 \mathrm{~h} 48$ min (SD $46 \mathrm{~min}$ ). The mean duration of each teaching exchange was $56 \mathrm{~s}$ (SD $46 \mathrm{~s}$, range $6 \mathrm{~s}$ to $4 \mathrm{~min} 25 \mathrm{~s}$ ), with a mean of seven teaching topics (SD 5.9 topics, range $0-27$ topics) across teams per day (see Table 1 for full distribution of rounding and teaching times). Duration of rounds was moderately positively correlated with daily teaching time $(r=0.32, p<0.01)$ and number of daily teaching topics $(r=0.50, p<0.01)$.
Teaching topics covered nearly all of the ABIM blueprint. In fact, only $5.6 \%$ (49) of topics observed could not be mapped. Table 2 shows the distribution of topics across the ABIM blueprint.

Cluster Analysis. Analysis revealed clustering in three distinct patterns (Fig. 1; low patient complexity/high clinical workload, average patient complexity/low clinical workload, and high patient complexity/high clinical workload). Specifically, teams 5,7 , and 8 were categorized into a single low patient complexity/high clinical workload cluster; teams $2,3 \mathrm{a}, 4 \mathrm{~b}, 9$, and 10 formed an average patient complexity/low clinical workload cluster; and teams $1,3 \mathrm{~b}, 4 \mathrm{a}$, and 6 were a high patient complexity/ high clinical workload cluster. Table 3 contains patient complexity variables, clinical workload variables, and educational measures, organized by cluster. 
Table 1 Daily Time Spent Teaching, Number of Topics Discussed, and Teaching Per Topics Discussed Including Daily Rounding Time

\begin{tabular}{|c|c|c|c|c|c|c|c|c|c|}
\hline \multirow[t]{2}{*}{ Team } & \multicolumn{3}{|c|}{$\begin{array}{l}\text { Daily Time Spent Teaching Per } \\
\text { Topic } \\
\text { (MM:SS) }\end{array}$} & \multicolumn{3}{|c|}{$\begin{array}{l}\text { Daily Time Spent Teaching } \\
\text { (MM:SS) }\end{array}$} & \multirow{2}{*}{$\begin{array}{l}\text { Daily Topics } \\
\text { Range }^{\dagger}\end{array}$} & \multicolumn{2}{|c|}{$\begin{array}{l}\text { Daily Rounding Time } \\
\text { (HH:MM:SS) }\end{array}$} \\
\hline & $\mathbf{N}_{\text {obs }} *$ & Mean (SD) & Range $^{\dagger}$ & Mean (SD) & Range $^{\dagger}$ & Mean (SD) & & Mean (SD) & Range $^{\dagger}$ \\
\hline 1 & 26 & $1: 21(1: 01)$ & $0: 15-4: 25$ & $6: 14(5: 25)$ & $0: 40-20: 18$ & $4.6(3.2)$ & $1-14$ & $1: 51: 05(34: 44)$ & $48: 56-2: 59: 15$ \\
\hline 2 & 26 & $0: 33(0: 40)$ & $0: 11-3: 02$ & $1: 45(2: 05)$ & $0: 22-9: 00$ & $2.8(2.5)$ & $1-11$ & $1: 54: 33(32: 38)$ & $56: 10-3: 13: 58$ \\
\hline $3 a$ & 15 & $0: 59(0: 22)$ & $0: 26-1: 34$ & $8: 39(5: 16)$ & $1: 26-21: 15$ & $9.8(6.7)$ & $1-26$ & $1: 45: 56(35: 47)$ & $44: 34-2: 27: 25$ \\
\hline $3 b$ & 11 & $0: 43(0: 23)$ & $0: 17-1: 19$ & $4: 22(3: 45)$ & $1: 30-13: 10$ & $6.1(2.8)$ & $2-10$ & $1: 38: 41(35: 39)$ & $50: 31-2: 24: 18$ \\
\hline $4 a$ & 15 & $0: 56(0: 41)$ & $0: 06-3: 24$ & $2: 38(2: 24)$ & $0: 06-8: 52$ & $3.5(3.4)$ & $1-13$ & $1: 59: 54(01: 16)$ & $30: 33-4: 54: 09$ \\
\hline $4 b$ & 11 & $0: 34(0: 18)$ & $0: 24-1: 00$ & $1: 59(1: 14)$ & $1: 00-3: 28$ & $4.0(2.4)$ & $1-7$ & $1: 37: 04(27: 52)$ & $52: 57-2: 15: 28$ \\
\hline 5 & 21 & $0: 46(0: 54)$ & $0: 09-3: 52$ & $5: 42(5: 15)$ & $0: 09-16: 27$ & $7.4(4.8)$ & $1-17$ & $2: 27: 48(28: 38)$ & $1: 41: 58-3: 30: 10$ \\
\hline 6 & 26 & $0: 43(0: 27)$ & $0: 17-2: 00$ & $9: 57(5: 04)$ & $1: 39-21: 32$ & $15.9(7.2)$ & $3-27$ & $2: 31: 23(1: 03: 30)$ & $27: 10-4: 50: 27$ \\
\hline 7 & 24 & $0: 57(0: 35)$ & $0: 10-3: 57$ & $7: 11(8: 08)$ & $0: 10-23: 08$ & $7.2(5.0)$ & $1-18$ & $1: 25: 21(30: 23)$ & $27: 46-2: 14: 07$ \\
\hline 8 & 25 & $1: 02(0: 58)$ & $0: 12-3: 17$ & $5: 58(6: 29)$ & $0: 26-22: 49$ & $5.1(2.4)$ & $1-9$ & $1: 20: 03(19: 51)$ & $43: 00-1: 56: 43$ \\
\hline 9 & 30 & $0: 32(0: 26)$ & $0: 08-2: 10$ & $4: 52(4: 55)$ & $0: 22-19: 28$ & $8.6(5.6)$ & $1-20$ & $1: 38: 48(46: 28)$ & $25: 13-2: 59: 57$ \\
\hline 12 & 14 & $0: 58(0: 42)$ & $0: 15-2: 20$ & $4: 53(6: 46)$ & $0: 15-25: 37$ & $4.1(3.3)$ & $1-11$ & $1: 26: 39(41: 26)$ & $20: 36-2: 29: 09$ \\
\hline Total & 244 & $0: 56(0: 46)$ & $0: 06-4: 25$ & $5: 59(5: 39)$ & $0: 06-25: 37$ & $7.0(5.9)$ & $1-27$ & $1: 48: 25(46: 38)$ & $20: 36-4: 54: 09$ \\
\hline
\end{tabular}

*Team rounds were not observed on weekends/holidays that team rounds did not occur, but instead attendings discussed patients with residents by phone. Explicit teaching occurred on 202 of the 244 days of observation

${ }^{\dagger}$ Minimums within ranges do not include days on which explicit teaching did not occur

\section{Total Teaching Time}

Together, team characteristics, including clinical workload (high vs. low) and patient complexity (high, average, or low), accounted for $9.1 \%$ of the variance in the total time spent teaching during any one patient's admission $(F[8,549]=6.90$, $p<0.001$ ). In particular, patient complexity (Average Comorbidities and Total Complications) across a patient's entire admission accounted for the majority of that explained variance $(7.8 \%)$ in total teaching time per patient $(\Delta F[2,549]=22.133, p<0.001$.

Measures of team workload and patient complexity were significantly associated with total time spent teaching. Specifically, high patient complexity/high clinical workload teams spent approximately $56 \mathrm{~s}$ longer teaching over the course of a patient's hospital stay than average patient complexity/low clinical workload teams (95\% CI: 11.74, 100.02). However, low patient complexity/high clinical workload teams did not differ significantly from the other two clusters in the amount of time spent teaching per patient.

Finally, with all other variables held constant, every time a patient experienced a complication during another patient's admission, the total time spent teaching for the patient without the complication decreased by almost $78 \mathrm{~s}$ on average $(95 \%$

Table 2 American Board of Internal Medicine Categories

\begin{tabular}{|c|c|c|c|}
\hline Allergy/immunology & $<1 \%(4)$ & Neurology & $4.6 \%(40)$ \\
\hline $\begin{array}{l}\text { Cardiovascular } \\
\text { disease }\end{array}$ & $13.0 \%(113)$ & $\begin{array}{l}\text { Obstetrics and } \\
\text { gynecology }\end{array}$ & $<1 \%(3)$ \\
\hline Dermatology & $<1 \%(4)$ & Oncology & $3.2 \%(27)$ \\
\hline $\begin{array}{l}\text { Endocrinology, DM, } \\
\text { and metabolism }\end{array}$ & $5.6 \%$ (49) & Ophthalmology & $<1 \%(3)$ \\
\hline Gastroenterology & $13.3 \%(116)$ & $\begin{array}{l}\text { Otolaryngology } \\
\& \text { dental }\end{array}$ & $<1 \%(2)$ \\
\hline Geriatrics & $4.0 \%(35)$ & Psychiatry & $2.6 \%(23)$ \\
\hline Hematology & $7.6 \%(66)$ & $\begin{array}{l}\text { Pulmonary } \\
\text { disease }\end{array}$ & $8.7 \%(76)$ \\
\hline Infectious disease & $16.0 \%(139)$ & $\begin{array}{l}\text { Rheumatology } \\
\text { \& orthopedics }\end{array}$ & $4.4 \%(38)$ \\
\hline $\begin{array}{l}\text { Nephrology \& } \\
\text { urology }\end{array}$ & $9.4 \%(82)$ & Other & $5.6 \%(49)$ \\
\hline
\end{tabular}

DM, diabetes mellitus
CI: $-142.21,-13.35)$. On the other hand, each additional complication experienced by a patient during his or her admission increased the total time spent teaching for that patient by 86 s (95\% CI: 59.14, 113.16).

\section{Total Teaching Topics}

Measures of team workload and patient complexity accounted for $16 \%$ of the variance in the number of teaching topics discussed by physicians during a patient's admission $(F[8,548]=14.18, p<0.001)$.

Specifically, high patient complexity/high clinical workload teams discussed approximately three more teaching topics per patient than low patient complexity/high clinical workload teams (95\% CI: $-4.48,-1.68)$, or approximately one more teaching topic per patient than average patient complexity/low clinical workload teams (95\% CI: 0.47, 1.97). Average patient complexity/low clinical workload teams discussed approximately two more teaching topics per patient than low patient complexity/ high clinical workload teams (95\% CI: $-3.18,-0.56)$.

Additionally, a one-unit increase (defined as one standard deviation) in the average daily complications of all other patients seen during a patient's admission decreased the total number of teaching topics discussed for that patient by almost three topics on average (95\% CI: $-3.70,-1.67)$. Conversely, each additional complication experienced by a patient during his or her admission increased the total number of teaching topics discussed for that patient by almost two topics (95\% CI: 1.63, 2.55).

\section{DISCUSSION}

This study represents a novel prospective assessment of the relationships between clinical workload, patient complexity, and teaching. We found that teaching, in terms of both the number of topics and the time spent per topic, varied significantly between teams. We observed teams in which relatively 
Table 3 Clinical Workload, Patient Complexity, and Teaching Measures by Complexity/Workload Clusters

\begin{tabular}{|c|c|c|c|}
\hline & $\begin{array}{l}\text { Low } \\
\text { Complexity, } \\
\text { High } \\
\text { Workload } \\
\text { Teams 5, 7, 8 }\end{array}$ & $\begin{array}{l}\text { Average } \\
\text { Complexity, } \\
\text { Low } \\
\text { Workload } \\
\text { Teams 2, 3a, } \\
\text { 4b, 9, 10 }\end{array}$ & $\begin{array}{l}\text { High } \\
\text { Complexity, } \\
\text { High } \\
\text { Workload } \\
\text { Teams 1, 3b, } \\
\text { 4a, } 6\end{array}$ \\
\hline Clinical Workload & Mean (SD) & Mean (SD) & Mean (SD) \\
\hline Daily Census & $13.19(2.98)$ & $10.14(4.03)$ & $12.95(3.52)$ \\
\hline Daily Turnover & $4.96(2.97)$ & $3.56(2.55)$ & $3.86(3.16)$ \\
\hline $\begin{array}{l}\text { Average Daily } \\
\text { Census during } \\
\text { each patient's } \\
\text { hospitalization }\end{array}$ & $13.70(2.74)$ & $10.69(3.55)$ & $13.57(2.45)$ \\
\hline $\begin{array}{l}\text { Turnover } \\
\text { occurring daily } \\
\text { during each } \\
\text { patient's } \\
\text { hospitalization }\end{array}$ & $3.75(0.35)$ & $3.40(.65)$ & $3.46(.59)$ \\
\hline \multicolumn{4}{|l|}{ Patient Complexity } \\
\hline $\begin{array}{l}\text { Comorbidity } \\
\text { Score for each } \\
\text { patient on } \\
\text { admission }\end{array}$ & $4.50(2.80)$ & $6.64(0.90)$ & $6.62(2.80)$ \\
\hline $\begin{array}{l}\text { Comorbidities of } \\
\text { other patients } \\
\text { hospitalized } \\
\text { during each } \\
\text { patient's } \\
\text { hospitalization }\end{array}$ & $4.42(.72)$ & $6.64(3.07)$ & $7.04(0.57)$ \\
\hline $\begin{array}{l}\text { Average } \\
\text { Comorbidities } \\
\text { of patients } \\
\text { each day }\end{array}$ & $4.57(.67)$ & $6.77(.89)$ & $7.17(0.82)$ \\
\hline $\begin{array}{l}\text { Daily } \\
\quad \text { Complications }\end{array}$ & $0.40(.81)$ & $0.83(0.98)$ & $0.99(1.12)$ \\
\hline $\begin{array}{l}\text { Total } \\
\text { Complications* }\end{array}$ & $0.15(.39)$ & $0.40(0.78)$ & $0.34(0.65)$ \\
\hline $\begin{array}{c}\text { Complications of } \\
\text { other patients }\end{array}$ & $0.31(.07)$ & $0.76(0.40)$ & $0.86(0.18)$ \\
\hline \multicolumn{4}{|c|}{ Teaching measures by patient } \\
\hline $\begin{array}{l}\text { Total time spent } \\
\text { teaching each } \\
\text { day }\end{array}$ & $6.26(6.73)$ & $4.30(5.00)$ & $6.43(5.30)$ \\
\hline $\begin{array}{l}\text { Total number of } \\
\text { topics } \\
\text { discussed each } \\
\text { day }\end{array}$ & $5.73(4.47)$ & $5.42(5.41)$ & $8.06(7.29)$ \\
\hline $\begin{array}{l}\text { Total time spent } \\
\text { teaching per } \\
\text { patient }\end{array}$ & $1.77(3.31)$ & $1.84(3.37)$ & $2.36(3.65)$ \\
\hline $\begin{array}{l}\text { Total number of } \\
\text { topics } \\
\text { discussed per } \\
\text { patient }\end{array}$ & $1.62(2.37)$ & $2.28(3.26)$ & $3.00(4.97)$ \\
\hline
\end{tabular}

*Total Complications is the number of complications a patient experienced over the course of his or her hospitalization

${ }^{+}$Complications of other patients is the average number of complications per day that developed in all of the other patients on a team while that team cared for a given patient

little explicit teaching occurred on rounds. Unsurprisingly, there was a moderate positive correlation between length of rounds and both daily teaching time and number of teaching topics. Clinical workload and patient complexity accounted for a modest, albeit significant, variance in teaching time and teaching topics. This tempers concerns about a simple negative relationship between workload and teaching.

Unanticipated patient complications also led to changes in teaching. We observed that, in the case of a patient with a complication, the time spent teaching increased, as did the number of teaching topics across that patient's hospitalization. Also unsurprisingly, reciprocal negative trends were seen with teaching time and topics with other patients on the team. Although these associations were small, they reflect the dynamic nature of teaching and demonstrate how teaching and advancing clinical care often occur simultaneously, and how the care (and related teaching) of one patient can influence that of others.

Despite the dynamic and largely unscripted nature of teaching on the wards, teaching topics were varied, nearly reaching saturation on the ABIM blueprint. While multiple factors influence teaching time and topics, general medicine wards remain a comprehensive teaching venue for core internal medicine content.

Our findings add to the literature describing contemporary rounds. Crumlish et al. provided detailed descriptions of the rounding activities of a single-center hospitalist service, noting that teaching rounds averaged $100 \mathrm{~min} .{ }^{1}$ Another observational study, which also included pediatric services, found a similar mean length (104.8 min) of attending rounds, of which an average of $10.3 \mathrm{~min}$ was spent on dedicated "educational activities." 18 Our findings were similar to these studies, with a mean $108 \mathrm{~min}$ of rounding time and $6 \mathrm{~min}$ of dedicated teaching.

We expected clinical workload and patient complexity to be associated with reductions in teaching time and topics covered. However, we were surprised that associations between workload characteristics, patient complexity, and teaching measures were relatively weak, and the associations that were found were not in the expected direction. A number of factors may have influenced our findings.

First, it is important to revisit how we defined and measured teaching. We both defined and measured teaching by measuring time spent and by number of topics. While practical and precise, these measurements did not capture all of the teaching and learning intertwined in patient discussions. While explicit teaching correlated only modestly with clinical workload and patient complexity, the richness of patient discussions might be impacted by these factors. Informal and tacit knowledge related to patient care, professionalism, and communication were not necessarily captured. Thus, it is possible that quality of teaching (and ultimately of learning) is impacted more by factors such as clinical workload and patient complexity, but this was outside the scope of our study.

Also, we defined our teams by attendings (i.e., when attendings changed, our team designation changed). While this is a reasonable definition, individual faculty characteristics may have accounted for significant variance. It is widely accepted that there is a range of teaching ability, interest, and devotion among attendings. Characteristics of effective clinical teachers have been described, ${ }^{19}$ and certainly individual idiosyncrasies related to teaching may have affected our results. A related possibility is that team characteristics, or the social relationships within teams, were important. Our previous work has demonstrated that relationships within teams are associated with improved patient outcomes. ${ }^{15}$ It is not much of a stretch to envision these relationships also affecting learning climate and teaching outcomes. 
While our teams were defined by attendings, team clusters were organized by clinical workload and patient complexity measures. Thus, clusters were defined by external factors independent of individual team membership. Our study was not designed to answer the question of how teams with more teaching would respond to increased clinical workload and patient complexity. Instead, we can only say that across teams, these external factors had only a modest effect on teaching time and topics. Following the same attendings over the course of an academic year would get to the root of this question.

There were limitations to our study. We only examined a single, large, university-based residency program at two teaching hospitals. Thus, our findings may not be broadly applicable to smaller or community-based residency programs. However, our observations were robust, with over $370 \mathrm{~h}$ of rounds observed. Our data were collected by observation, and a Hawthorne effect is possible. That said, our experience observing these teams was that, given the quantity of work to do, they quickly acclimated to our presence (within 1-2 days) and settled into their rounding routines. Also, our observations took place over 6 years. While potential temporal changes in workload and teaching could have affected our results, we feel that our workload and teaching practices did not change dramatically during this time frame. Finally, we did not capture time spent teaching outside of rounds in this analysis. While we are aware that there was teaching outside of rounds, this was variable and was largely decontextualized from patient care.

\section{CONCLUSION}

Our study adds to the literature describing contemporary teaching rounds. While there was a small correlation observed between clinical workload, patient complexity, and teaching, our findings suggest that the relationship between these variables and the quantity of teaching is more complex than anticipated. Future work should confirm that this relationship is indeed modest, while also examining the impact of clinical workload and patient complexity on quality of teaching. In addition, future studies should search for other factors that may influence educational quality on teaching services.

\section{Acknowledgements:}

We would like to thank the members of the teams involved in this study.

\section{Contributors: None.}

Prior Presentations: None.

Corresponding Author: Temple A. Ratcliffe, MD; University of Texas Health Science Center at San Antonio, 7703 Floyd Curl Drive, San Antonio, TX 78229-3900, USA (e-mail: ratcliffe@uthscsa.edu).

Funders The research reported here was supported by the Department of Veterans Affairs, Veterans Health Administration, Health Services Research and Development Service (CDA 07-022).
Investigator salary support is provided through this funding, and through the South Texas Veterans Health Care System. The views expressed in this article are those of the authors and do not necessarily reflect the position or policy of the Department of Veterans Affairs.

\section{Compliance with Ethical Standards:}

Conflict of Interest: The authors declare that they do not have a conflict of interest.

\section{REFERENCES}

1. Crumlish CM, Yialamas MA, McMahon GT. Quantification of bedside teaching by an academic hospitalist group. J Hosp Med. 2009;4(5):304-7.

2. Stickrath C, Noble M, Prochazka A, Anderson M, Griffiths M, Manheim J, Sillau S, Aagaard E. Attending rounds in the current era: what is and is not happening. JAMA Intern Med. 2013;173(12):1084-9.

3. Shoeb M, Khanna R, Fang $\mathbf{M}$, Sharpe B, Finn $\mathbf{K}$, Ranji S, Monash B. Internal medicine rounding practices and the Accreditation Council for Graduate Medical Education core competencies. J Hosp Med. 2014;9(4):239-43.

4. Accreditation Council for Graduate Medical Education. Common Program Requirements. http://www.acgme.org/Portals/0/PDFs/Common_ Program_Requirements_07012011[2].pdf. Accessed December 19, 2017.

5. O'Conner AB, Lang VL, Bordley DR. Restructuring an inpatient resident service to improve outcomes for residents, students and patients. Acad Med 2011;86(12):1500-7.

6. Elliott DJ, Young RS, Brice J, Aguiar R, Kolm P. Effect of hospitalist workload on the quality and efficiency of care. JAMA Intern Med. 2014;174(5):786-93.

7. Kripalani S, Pope AC, Rask K, Hunt K, Dressler DD, Branch WT, Zhang R, Williams MV. Hospitalists as teachers. J Gen Intern Med. 2004;19(1):8-15.

8. Saint S, Flanders SA. Hospitalists in teaching hospitals: opportunities but not without danger. J Gen Intern Med. 2004;19(4):392-3.

9. Tierney MJ, Pageler NM, Kahana M, Pantaleoni JL, Longhurst CA. Medical education in the electronic medical record (EMR) era: benefits, challenges, and future directions. Acad Med. 2013;88(6):748-52.

10. Reilly JB, Bennett N, Fosnocht K, Williams K, Kangovi S, Jackson R, Ende J. Redesigning rounds: towards a more purposeful approach to inpatient teaching and learning. Acad Med. 2015;90(4):450-3.

11. McGee S. A piece of my mind. Bedside teaching rounds reconsidered. JAMA. 2014;311(19):1971-2.

12. Gonzalo JD, Chuang $\mathbf{C H}$, Huang G, Smith C. The return of bedside rounds: an educational intervention. J Gen Intern Med. 2010;25(8):792-8.

13. Leykum LK, Chesser H, Lanham HJ, Carla P, Palmer R, Ratcliffe T, Reisinger H, Agar M, Pugh J. The Association Between Sensemaking During Physician Team Rounds and Hospitalized Patients' Outcomes. J Gen Intern Med. 2015;30(12):1821-7.

14. Leykum LK, Lanham HJ, Provost SM, McDaniel RR, Pugh J. Improving outcomes of hospitalized patients: the Physician Relationships, Improvising, and Sensemaking intervention protocol. Implement Sci. 2014;9: 171.

15. McAllister C, Leykum LK, Lanham H, Reisinger HS, Kohn JL, Palmer R, Pezzia C, Agar M, Parchman M, Pugh J, McDaniel RR Jr. Relationships within inpatient physician housestaff teams and their association with hospitalized patient outcomes. J Hosp Med. 2014;9(12):764-71.

16. American Board of Internal Medicine. Internal Medicine Certification Examination Blueprint. https://www.abim.org/ /media/ ABIM\%20Public/Files/pdf/exam-blueprints/certification/internal-medicine.pdf Accessed December 19, 2017.

17. Deyo RA, Cherkin DC, Ciol MA. Adapting a clinical comorbidity index for use with ICD-9-CM administrative databases. J Clin Epidemiol. 1992;45(6):613-9.

18. Priest JR, Bereknyei S, Hooper K, Braddock CH 3rd. Relationships of the location and content of rounds to specialty, institution, patientcensus and team size. PLoS One. 2010;5(6):e11246.

19. Goldszmidt M, Faden L, Dornan T, van Merriënboer J, Bordage G, Lingard L. Attending physician variability: a model of four supervisory styles. Acad Med. 2015;90(11): 1541-6. 